\title{
An Emergence of Equine-Like G3P[8] Rotaviruses Associated with Acute Gastroenteritis in Hospitalized Children in Thailand, 2016-2018
}

\author{
Thanakorn Chaiyaem ${ }^{1}$, Chulapong Chanta ${ }^{2}$, and Wisoot Chan-it ${ }^{1}$ * \\ ${ }^{1}$ Biology Program, Faculty of Science and Technology, Pibulsongkram Rajabhat University, Phitsanulok 65000, Thailand \\ ${ }^{2}$ Pediatric Unit, Chiangrai Prachanukroh Hospital, Chiang Rai 57000, Thailand
}

Received: October 4, 2020 / Revised: November 17, 2020 / Accepted: November 23, 2020

\begin{abstract}
Rotavirus A (RVA) is recognized as a major etiology responsible for the development of acute gastroenteritis in children worldwide. The purpose of the present study was to perform the molecular characterization of RVA. A total of 323 stool specimens collected from hospitalized children with acute gastroenteritis in Chiang Rai, Thailand, in 2016-2018 were identified for G- and P-genotypes through RT-PCR analysis. RVA was more prevalent in 2017-2018 (37.8\%) than in 2016-2017 (23.2\%). The seasonal peak of RVA occurred from March to April. G3P [8] was predominant in 2016-2017 (90.6\%) and 2017-2018 (58.6\%). Other genotypes including G1P[8], G8P[8], G9P[8], and mixed infections were also identified. G3P[8] strains clustered together in the same lineage with other novel human equine-like G3P[8] strains previously identified in multiple countries and presented a genotype 2 constellation (G3-P[8]-I2-R2-C2-M2-A2-N2-T2-E2-H2). Several amino acid differences were observed in the antigenic epitopes of the VP7 and VP8* capsid proteins of the equine-like G3P[8] compared with those of the RVA vaccine strains. The homology modeling of the VP7 and VP8* capsid proteins of the equine-like G3P[8] strains evidently exhibited that these residue differences were present on the surface-exposed area of the capsid structure. The emergence of the equine-like G3P[8] strains in Thailand indicates the rapid spread of strains with human and animal gene segments. Continuous surveillance for RVA is essential to monitor genotypes and genetic diversity, which will provide useful information for selecting rotavirus strains to develop a safe and effective RVA vaccine that is efficacious against multiple genotypes and variants.
\end{abstract}

Keywords: Rotavirus, acute gastroenteritis, epidemiology, genotyping, equine-like G3P[8], homology modeling

\section{Introduction}

Rotavirus A (RVA) is recognized as a major worldwide etiologic agent causing acute gastroenteritis in young children and different animal species. In humans, RVA infection can lead to high morbidity and mortality for patients, who develop severe dehydration, contributing an estimated 215,000 deaths among the children aged $<5$ years annually [1]. RVA is classified into the Reoviridae family, Sedoreovirinae subfamily, and Rotavirus genus.

\section{* Corresponding author}

Tel: +66-82-4019079, Fax: +66-55-267104

E-mail:wchanit@psru.ac.th
Genomes of RVA comprise 11 segments of dsRNA, which are surrounded by a triple-layered and non-enveloped capsid of icosahedral symmetry. The genomes encode 6 structural proteins (VP1 to VP4, VP6, and VP7) and 5 or 6 non-structural proteins (NSP1 to NSP5 or NSP6). VP7 (G antigen, glycoprotein) and VP4 (P antigen, protease sensitive) are the outermost capsid layers, which function as the virus cell-attachment proteins and independently elicit neutralizing antibodies. Genetically, VP7 is classified into G-types and VP4 into P-types. Full genome analysis using the convention Gx-P[x]-Ix-Rx-CxMx-Ax-Nx-Tx-Ex-Hx, which represents genotypes of VP7-VP4-VP6-VP1-VP2-VP3-NSP1-NSP2-NSP3-NSP4- 
NSP5/NSP6, respectively, is required to determine the evolutionary pattern through the reassortment event of RVA [2]. Currently, 36 G, 51 P, 26 I, 22 R, 20 C, 20 M, $31 \mathrm{~A}, 22 \mathrm{~N}, 22 \mathrm{~T}, 27 \mathrm{E}$, and $22 \mathrm{H}$ types have been reported for exiting human and animal rotaviruses (https://rega.kuleuven.be/cev/viralmetagenomics/virusclassification/rcwg). G1P [8], G2P [4], G3P [8], G4P [8], and G9P[8] are considered as common genotypes to cause diarrhea in humans [3].

Genetic diversity of RVA can occasionally evolve through the process of intergenotype reassortment events during co-infection between RVA of human and animal origins, resulting in the increased detections of RVA bearing unusual genotypes. Since 2013, the rapid geographic distribution among human population of unusual equine-like G3 intergenogroup reassortant rotaviruses in association with $\mathrm{P}[4], \mathrm{P}[6]$, or $\mathrm{P}[8]$ have been detected in many countries, including Australia [4-6], Brazil [7-9], Dominican Republic [10], Germany [11], Hungary [12], Indonesia [13-15], Ireland [16], Italy [17], Japan [1821], Spain [22], Thailand [23, 24], and USA [25].

First surveillance of RVA in Chiang Rai province of Thailand has previously described the finding an emergence of G9P [8] as the predominant genotype in 20152016 [23]. In order to continue monitoring of circulating RVA in Chiang Rai, the purpose of the present study was the molecular characterization of RVA in Chiang Rai, Thailand, 2016-2018.

\section{Materials and Methods}

\section{Patients and specimen collection}

A total of 323 fecal specimens were collected from infants and children with acute gastroenteritis admitted to Chiangrai Prachanukroh Hospital in Chiang Rai, Northern Thailand. The specimen collection period was from March 2016 to February 2018 (a two-year period). The ages of the subjects ranged from neonate up to 5 years old. Acute gastroenteritis was defined as the occurrence of 3 or more episodes of watery or looser than nor$\mathrm{mal}$ stool in a $24 \mathrm{~h}$ period before the visit. The specimens were stored at $-20^{\circ} \mathrm{C}$ until used.

\section{Detection of RVA by RT-PCR}

Viral RNAs were extracted from $10 \% \mathrm{w} / \mathrm{v}$ fecal suspensions using Viral Nucleic Acid Extraction Kit II
(Geneaid), according to the manufacturer's instructions. The extracted RNAs were reverse transcribed to synthesize cDNAs by reverse transcription using ReverTra Ace ${ }^{\circledR}$ qPCR-RT Master Mix (Toyobo). RVA was detected by RT-PCR using 5x HOT FIREPol Blend Master Mix (Solis BioDyne) with Beg9/VP7-1' primer pair, yielding a 395-bp amplicon [26].

\section{Genotype identification of RVA}

RVA-positive samples were G- and P-genotyped by semi-nested multiplex PCR assays. Briefly, the firstround PCR was performed using conserved primers Beg9/End9 or VP7-F/VP7-R for VP7 gene and Con3/Con2 for VP4 gene. The second-round semi-nested multiplex PCR was performed using G-type-specific primers: G1G4, G9, and G12; and P-type-specific primers: P [4], P [6], and P[8] [27-29]. For untypable samples, multiplexed one-step genotyping method with new G- and P-target specific primers was performed to identify G1-G4, G9, and G12; and P[4], P[6], P[8], P[9], and P[10] [30]. For those samples that still remained untyped were subjected to nucleotide sequencing.

Some of RVA-positive samples that had been successfully typed as G3 by the method mentioned above were selected as representatives for each month for further PCR to confirm whether they were an equine-like G3 genotype using the equine-like G3 specific primers EQG3FWD 5'-CTGCATACGCTAATTCTACACAAGG-3' and EQG3REV 5'-GATCGTACAAGTAGCCGTAGTAA C-3', which amplified a 544-bp amplicon of the VP7 gene of the equine-like G3 [4].

G- and P-genotypes were examined by electrophoresis using 1.5\% agarose gel, stained with RedSafe ${ }^{\mathrm{TM}}$ Nucleic Acid Staining Solution (20000x) (iNtRON Biotechnology, Korea), and observed under UV light.

\section{Sequence analyses of the equine-like G3P8] strains}

The 11 genomes of the equine-like G3P[8] strains were partially amplified by PCR using 2x Long Range Master Mix (Biotechrabbit, Germany). Primers used in the amplification were published elsewhere [31]. PCR amplicons were purified using Gel/PCR DNA Fragments Extraction Kit (Geneaid), and then sequenced using BigDye Terminator Cycle Sequencing Kit (Applied Biosystems, USA) in an automated ABI Prism 310 Genetic Analyzer (Applied Biosystems). Multiple alignments of 
nucleotide and amino acid sequences between studied and reference strains were analyzed. Phylogenetic trees, based on the partial nucleotide sequences of VP1 (985 bp), VP2 (918 bp), VP3 (859 bp), VP4 (852 bp), VP6 (848 bp), VP7 (1,052 bp), NSP1 (811 bp), NSP2 (891 bp), NSP3 (870 bp), NSP4 (672 bp), and NSP5 (755 bp), were constructed by the neighbor-joining method in MEGA 7.0. The statistical significance of genetic relationship was estimated by bootstrap resampling analysis $(1,000$ replications). Nucleotide sequences of the analyzed RVA strains had been submitted to the GenBank under accession number MK161352-MK161391.

\section{Homology modeling of VP7 and VP8* capsid proteins of the equine-like $\mathbf{G} 3$ strains}

Homology modeling of VP7 and VP8* outer capsid proteins were constructed using the G3 VP7 trimer in complex with a neutralizing Fab (PDB: 3FMG) and the VP8* domain from a P[8] VP4 spike-forming protein binding to the secretory $\mathrm{H}$ type- 1 antigen (PDB: 6HA0) as templates, respectively, using the UCSF Chimera- and ChimeraX-Molecular Modeling systems. Briefly, the BLAST proteins between targets and templates, using the Chimera, demonstrated high amino acid sequence identity of $98 \%$ for VP7 and $97 \%$ for VP8*, indicating that the $3 \mathrm{FMG}$ and the $6 \mathrm{HA} 0$ were appropriate templates for generating homology models. For comparative modeling of the VP7, a monomer was first predicted by Chimera interface to Modeller and saved it to a PDB file for 3 copies with different names. The trimer of the VP7 model was then generated by superposition of each monomer model onto each chain in the trimeric structure of 3FMG.PDB1 biological assembly 1 using the program ChimeraX. The comparative VP8* model was only predicted and generated into a monomer form using the same method as above. Finally, the trimer form of con- structed VP7 models and the monomer form of VP8* models were visualized by Chimera; amino acid changes present on the exposed-surface area of the outer capsid structures were highlighted and labeled.

\section{Ethics}

The study protocol was conducted according to the approval by the Ethics Committee, Chiangrai Prachanukroh Hospital (CR 0032.102/34017 and CR 0032.102/ 15688). Parents or caretakers of pediatric patients were described by doctors and nurses about objectives and methods used for laboratory research. Permission for collecting stool specimens directly from diapers and clinical information of the patients was received from their parents who were asked to sign an informed consent. The clinical data included frequency of diarrhea (episodes/ day), vomiting (episodes/day) and body temperature.

\section{Results}

\section{Molecular epidemiology of RVA}

RVA was overall detected in 102 of 323 stool specimens (31.6\%): $23.2 \%$ in 2016-2017 and 37.8\% in 20172018 (Table 1). Most of the patients with RVA infection were in a 12 - to 23 -month-old group: $43.8 \%$ in $2016-$ 2017 and 34.3\% in 2017-2018 (Table 2). Patients under 36 months old or 3 years of age were greatly infected by RVA: $84.4 \%$ in 2016-2017 and 92.8\% in 2017-2018. RVA was detected for 4 months in 2016-2017 and almost all months in 2017-2018. The seasonal peak occurred during the summer from March to April: 90.7\% in 2016-2017 and 70\% in 2017-2018 (Table 3).

\section{Distribution of G- and P-genotypes of RVA}

G3P[8] was the predominant genotype: $90.6 \%$ in 2016-2017 and 58.6\% in 2017-2018. G9P[8] was identi-

Table 1. Detection and distribution of G- and P-genotype combinations of RVA in Chiang Rai, Thailand.

\begin{tabular}{|c|c|c|c|c|c|c|c|c|c|c|}
\hline \multirow{2}{*}{ Year } & \multirow{2}{*}{$\begin{array}{l}\text { No. of } \\
\text { samples }\end{array}$} & \multirow{2}{*}{$\begin{array}{l}\text { No. of RVA-positive } \\
\text { samples (\%) }\end{array}$} & \multicolumn{7}{|c|}{ No. of genotypes (\%) } & \multirow{2}{*}{ Reference } \\
\hline & & & G1P[8] & G3P[8] & G8P[8] & G9P[8] & G1,G3P[8] & G1,G9P[8] & G3,G9P[8] & \\
\hline 2015-2016 & 270 & $\begin{array}{c}91 \\
(33.7)\end{array}$ & $\begin{array}{c}3 \\
(3.3)\end{array}$ & $\begin{array}{c}12 \\
(13.2)\end{array}$ & 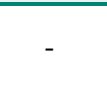 & $\begin{array}{c}72 \\
(79.1)\end{array}$ & $\begin{array}{c}2 \\
(2.2)\end{array}$ & $\begin{array}{c}1 \\
(1.1)\end{array}$ & $\begin{array}{c}1 \\
(1.1)\end{array}$ & [23] \\
\hline 2016-2017 & 138 & $\begin{array}{c}32 \\
(23.2)\end{array}$ & - & $\begin{array}{c}29 \\
(90.6)\end{array}$ & $\begin{array}{c}1 \\
(3.1)\end{array}$ & $\begin{array}{c}2 \\
(6.3)\end{array}$ & - & - & - & This study \\
\hline $2017-2018$ & 185 & $\begin{array}{c}70 \\
(37.8)\end{array}$ & $\begin{array}{c}7 \\
(10.0)\end{array}$ & $\begin{array}{c}41 \\
(58.6)\end{array}$ & - & $\begin{array}{c}20 \\
(28.6)\end{array}$ & - & - & $\begin{array}{c}2 \\
(2.8)\end{array}$ & This study \\
\hline
\end{tabular}


Table 2. Age-related distribution of patients infected with RVA in Chiang Rai, Thailand.

\begin{tabular}{cccc}
\hline \multirow{2}{*}{$\begin{array}{c}\text { Age group } \\
\text { (months) }\end{array}$} & \multicolumn{3}{c}{ No. of RVA-positive samples (\%) } \\
\cline { 2 - 4 } & $\begin{array}{c}2016-2017 \\
(\mathrm{n}=32)\end{array}$ & $\begin{array}{c}2017-2018 \\
(\mathrm{n}=70)\end{array}$ & $\begin{array}{c}\text { Total } \\
(\mathrm{n}=102)\end{array}$ \\
\hline$\leq 5$ & $1(3.1)$ & $5(7.1)$ & $6(5.9)$ \\
$\mathbf{6 - 1 1}$ & $\mathbf{9 ( 2 8 . 1 )}$ & $\mathbf{2 5 ( 3 5 . 7 )}$ & $\mathbf{3 4}(\mathbf{3 3 . 3})$ \\
$\mathbf{1 2 - 2 3}$ & $\mathbf{1 4 ( 4 3 . 8 )}$ & $\mathbf{2 4 ( 3 4 . 3 )}$ & $\mathbf{3 8 ( 3 7 . 3 )}$ \\
$24-35$ & $3(9.4)$ & $11(15.7)$ & $14(13.7)$ \\
$36-47$ & $4(12.5)$ & $4(5.7)$ & $8(7.8)$ \\
$\geq 48$ & $1(3.1)$ & $1(1.4)$ & $2(2)$ \\
\hline
\end{tabular}

Table 3. Monthly distribution of RVA-positive samples in Chiang Rai, Thailand.

\begin{tabular}{llll}
\hline \multirow{2}{*}{ Month } & \multicolumn{3}{c}{ No. of RVA-positive samples (\%) } \\
\cline { 2 - 4 } & $\begin{array}{c}2016-2017 \\
(\mathrm{n}=32)\end{array}$ & $\begin{array}{c}2017-2018 \\
(\mathrm{n}=70)\end{array}$ & $\begin{array}{l}\text { Total (\%) } \\
(\mathrm{n}=102)\end{array}$ \\
\hline Mar & $\mathbf{1 4 ( 4 3 . 8 )}$ & $\mathbf{1 7}(\mathbf{2 4 . 3})$ & $\mathbf{3 1 ( 3 0 . 4 )}$ \\
Apr & $\mathbf{1 5 ( 4 6 . 9 )}$ & $\mathbf{2 5 ( 3 5 . 7 )}$ & $\mathbf{4 0}(\mathbf{3 9 . 2 )}$ \\
May & $1(3.1)$ & $7(10)$ & $8(7.8)$ \\
Jun & - & $3(4.3)$ & $2(2)$ \\
Jul & - & $2(2.9)$ & $3(2.9)$ \\
Aug & - & - & - \\
Sep & - & $1(1.4)$ & $1(1)$ \\
Oct & - & $5(7.1)$ & $5(4.9)$ \\
Nov & - & $1(1.4)$ & $1(1)$ \\
Dec & - & $5(7.1)$ & $5(4.9)$ \\
Jan & - & - & - \\
Feb & $2(6.3)$ & $4(5.7)$ & $6(5.9)$ \\
\hline
\end{tabular}

fied as the second most prevalent genotype in 2017-2018 (28.6\%) (Table 1). Other G- and P-genotypes and mixed infections were also identified as summarized in Table 1. Some of the identified G3 samples had been confirmed as the equine-like G3 genotype by PCR: 34.5\% (10 of 29 samples) of 2016-2017 and 48.7\% (20 of 41 samples) of 2017-2018 (data not shown).

\section{Nucleotide sequence analyses of the 11 genes of the equine-like G3P[8] strains}

VP7 and VP4 genes of 11 G3P[8] strains as representatives were sequenced and analyzed. Among these 11 samples, 2 strains RVA/Human-wt/THA/2CR29/2016/ G3P[8] and RVA/Human-wt/THA/2CR41/2016/G3P[8] were subjected to further nucleotide sequence analysis of their 11 genes. Analysis found that all VP7 and VP4 nucleotide sequences shared high nucleotide identity (99-100\%) with each other. Phylogenetic tree showed that the VP7 genes of Chiang Rai G3 strains clustered in the same lineage with other novel equine-like G3 strains recently identified in different countries, including Australia, Brazil, Dominican Republic, Germany, Hungary, Indonesia, Ireland, Italy, Japan, Spain, Thailand, and USA. In contrast, the VP7 genes of the equine-like G3 strains clustered in different lineages with other typical G3 strains detected in the past of Thailand before 2015 (Fig. 1A). Phylogenetic tree of the VP4 genes revealed that all of the $11 \mathrm{P}[8]$ genes clustered in the same $\mathrm{P}[8]$ lineage, most closely related to those of the equine-like $\mathrm{P}[8]$ strains (Fig. 1B).

Analysis of genomic constellation on the 2 representative strains RVA/Human-wt/THA/2CR29/2016 and RVA/Human-wt/THA/2CR41/2016 resulted in genotype 2 constellation of the backbone genes (G3-P[8]-I2-R2-C2M2-A2-N2-T2-E2-H2). Phylogenetic trees of the VP1VP3, VP6, and NSP1-NSP5 genes of the representative strains demonstrated close genetic relatedness with other human equine-like G3P[8] strains (Figs. S1A-S1I).

\section{Amino acid sequence analysis and homology modeling of VP7 and VP8* capsid proteins}

In an attempt to understand the molecular basis of amino acid differences on an outer capsid VP7 protein and a spike VP8* protein, the antigenic epitopes of the representative equine-like G3 strains were compared with those of RVA vaccine strains. And comparative models of VP7 and VP8* proteins were constructed to demonstrate positions of the amino acid differences. As the results, the equine-like G3 strain represented by RVA/Human-wt/THA/2CR29/2016/G3P[8] contained 14 amino acid differences across the antigenic regions compared with the G1 Rotarix ${ }^{\mathrm{TM}}$ vaccine strain (Fig. 2A). When compared with the G3 strain of RotaTeq ${ }^{\mathrm{TM}}$ vaccine, 4 amino acid differences were observed: 1 of which occurred in $7-1 \mathrm{a}$ region (T87S) and 3 in $7-1 \mathrm{~b}$ region (N213T, K238D, and D242A). The homology models clearly presented that these amino acid differences were located on the surface-exposed area of the VP7 capsid structure (Fig. 2B).

For VP8* neutralizing epitope comparison, the equinelike $\mathrm{P}[8]$ strain had 8 amino acid differences across the 

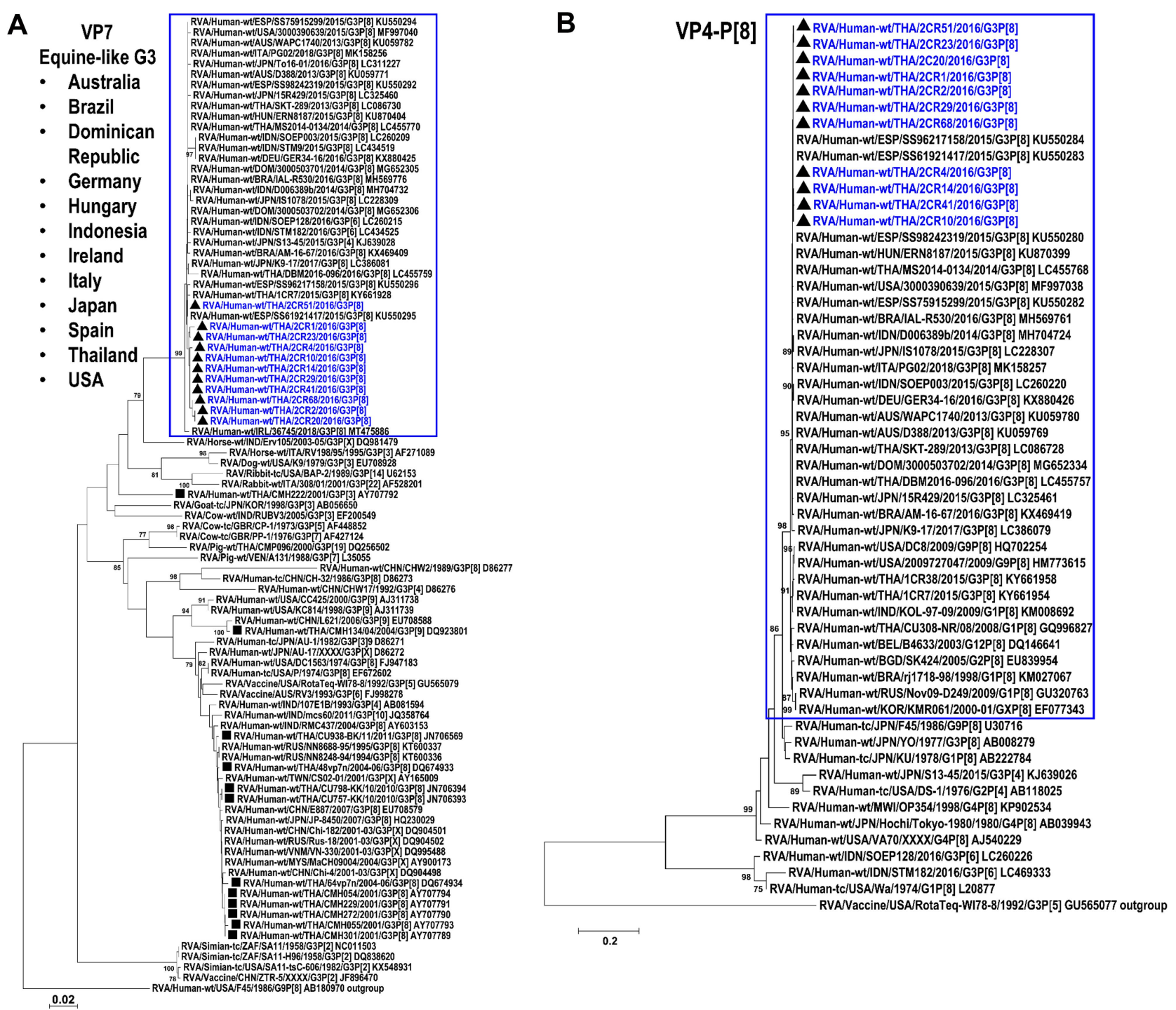

Fig. 1. Phylogenetic analyses of VP7 and VP4 genes of the equine-like G3P[8] strains. Phylogenetic trees were analyzed based on partial nucleotide sequences of (A) VP7 and (B) VP4 genes. RVA detected in the present study are labeled with $\boldsymbol{\Delta}$ in blue. Typical Thai G3 strains obtained from GenBank are labeled with $\mathbf{\square}$. The trees were constructed by neighbor-joining method and percent bootstrap support was indicated by the values at each node. The values less than 75 are omitted and the bar indicates the variation scale.

antigenic regions compared with the $\mathrm{P}[8]$ Rotarix ${ }^{\mathrm{TM}}$ vaccine strain (Fig. 3A), containing 8-1: S146G, N150D, S190N, and N196G; and 8-3: N113D, S125N, S131R, and N135D. The equine-like P8] strain comprised 4 amino acid differences: 8-1: S146G, N150S, and D196G; and 8-3: N113D) when compared with the P[8] RotaTeq ${ }^{\mathrm{TM}}$ vaccine strain. The VP8* comparative models also demonstrated that all of the amino acid differences were located on surface-exposed area of the spike VP8* protein (Fig. 3B).

\section{Discussion}

Acute gastroenteritis caused by RVA is a primary illness in children under 5 years of age and is considered as an important public health burden in both developing and developed countries. In this study, the follow-up surveillance of RVA was carried out by RT-PCR from diarrheal samples collected from hospitalized infants and children in Chiang Rai, Thailand, 2016-2018. RVA was recognized as the causative agent of the disease for 
A

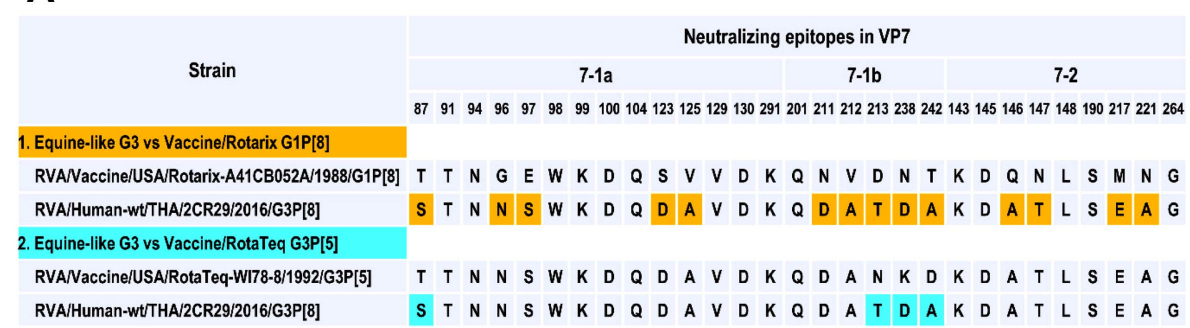

B
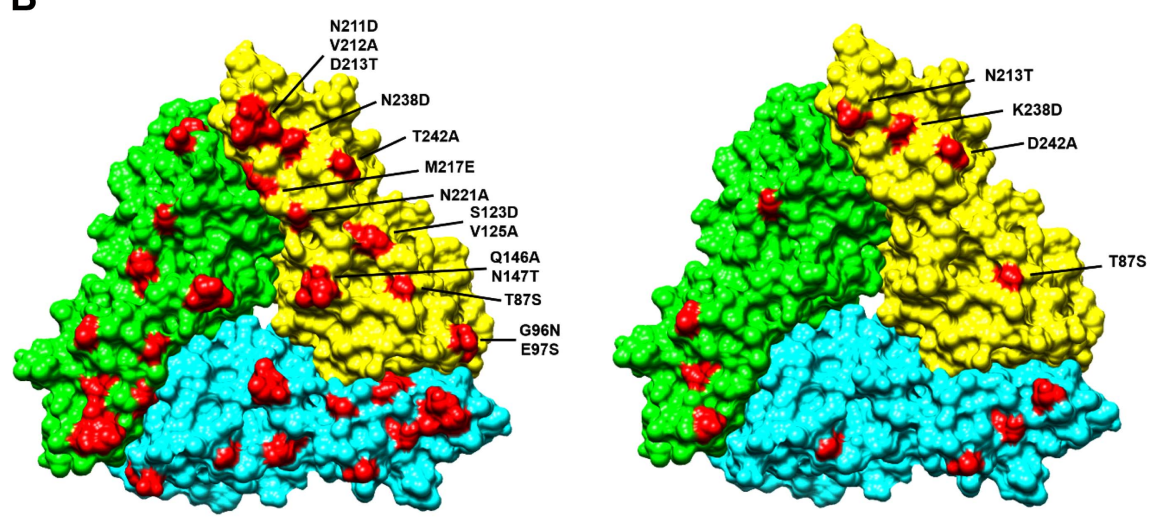

1. Equine-like $\mathrm{G} 3$ vs Vaccine/Rotarix $\mathrm{G} 1 \mathrm{P}[8]$

2. Equine-like G3 vs Vaccine/RotaTeq G3P[5]

Fig. 2. Amino acid comparison and homology modeling of the VP7 protein. (A) Antigenic regions of VP7 proteins were aligned, and compared between the equine-like G3 strain detected in this study and those of the 2 RVA vaccine strains. Residues highlighted in different colors are those that differ from the vaccine strains. (B) The VP7 homology models were constructed and surfaceexposed residues that differ from the RVA vaccine strains are in red.

$23.2 \%$ in $2016-2017$ and $37.8 \%$ in $2017-2018$ (Table 1), which is consistent with the results of previous reports in Thailand with RVA infection rates ranging from $17.8 \%$ to $44.5 \%$ [23, 24, 32-42].

Epidemiological reports had found that RVA infection more commonly occurred in children under 3 years of age $[23,32,43]$. In agreement with this, the present results showed a marked tread toward higher rates of infection in children under 3 years of age $(90.2 \%)$ versus children over 3 years of age $(9.8 \%)$ with children of the 12- to 23-month-old group being the most frequently affected (Table 2). The lower prevalence in older children could be described as they might develop acquired immunity through previous infection.

The seasonal peak of RVA infection in this surveillance appeared in summer between March and April (Table 3), which is in line with the finding of the previous season 2016-2017 in Chiang Rai [23] and other reports in Thailand [24, 44]. In addition, a different seasonal pattern of RVA infection in Thailand was also observed in rainy season between July and August with high detection rate and number of equine-like G3P [8] strains [24]. Maneekarn \& Khamrin [44] has summarized the seasonality of rotavirus gastroenteritis based on epidemiological researches in Thailand, which is in tropical region that rotavirus was detected all-yearround with the peak from November to April of the following year. In contrast to Japan, which is in temperate region, studies of non-hospitalized patients associated with RVA infection found that RVA peaked in spring during March to April, but was lowest during summer from July to August [43]. It has been hypothesized that rotavirus survival is favored by cooler conditions with low humidity in the temperate regions [45], but the smaller seasonal changes in temperature and humidity in some tropical settings compared to temperate ones could make it difficult to study weather as a driver of diarrheal disease, since weather covariates can be highly correlated (e.g., heat and humidity) [46]. In addition, different weather variables can also influence human 
A

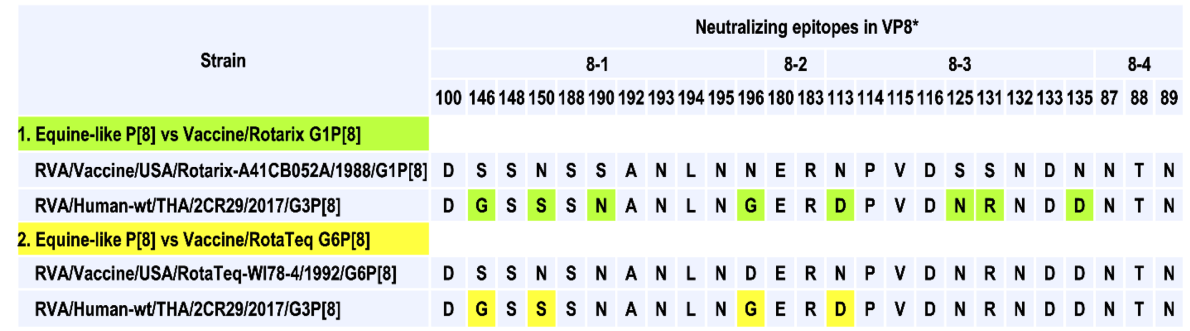

B
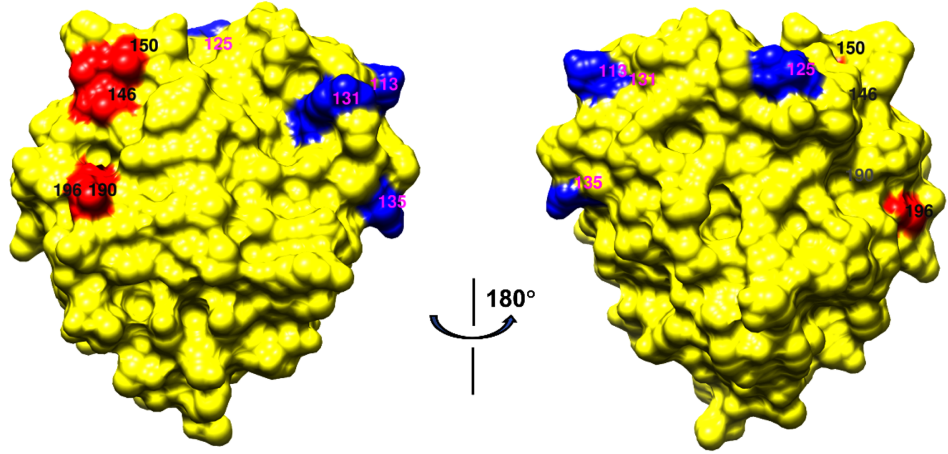

1. Equine-live P[8] vs Vaccine/Rotarix G1P[8]
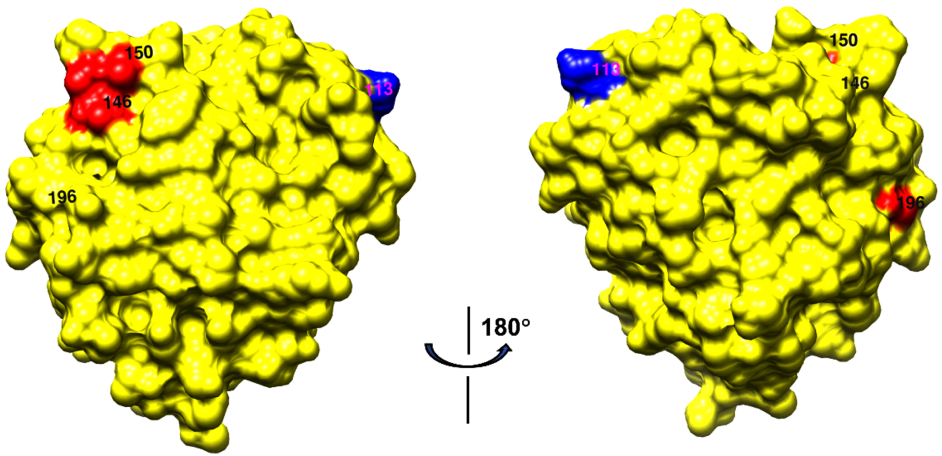

2. Equine-live P[8] vs Vaccine/RotaTeq G6P[8]

Fig. 3. Amino acid comparison and homology modeling of the VP8* protein. (A) Antigenic regions of VP8* proteins were aligned, and compared between the equine-like P[8] strain detected in this study and those of the 2 RVA vaccine strains. Residues highlighted in different colors are those that differ from the vaccine strains. (B) The VP8* homology models were constructed and surface-exposed residues that differ from the RVA vaccine strains are in red and blue.

behavior, sanitation, hygiene practices and the probability of environmental exposures [46]. For example, summer can encourage individuals to have outdoor or social activities, increasing the opportunity of close contact between infected and susceptible individuals, while rainfall can promote individuals to stay indoors. Taken together, seasonal fluctuation of RVA can vary by distinct geographical zones with different weather condition, such as temperature and humidity and can be influenced by other multiple factors, including route of transmission, social behaviors, health practices, meth- ods used to measure infection, and RVA genotypes [44, 45]. Knowledge derived from the seasonal trend of RVA infection in each region can be used to target vaccination program, test efficacy of the vaccine implementation program, and the vaccine itself [47].

Regarding $\mathrm{G}$ and $\mathrm{P}$ genotyping of RVA, 3 common G-P combinations comprising of G1P[8], G3P [8], and G9P[8] were identified in up to $97 \%$ of the studies samples. According to extensive epidemiological studies of RVA infection in Thailand since 2000, G1 is the most frequent genotype, followed by G9 [23, 32-38, 40-42]. RVA G3 
emerged as the leading genotype only in 2009-2011 [39]. In this study, interestingly, the distribution pattern of RVA in Chiang Rai has shifted from the previous season from G9P[8] [23] to G3P[8] (Table 1). G3P[8] in this study had similar genetic relatedness with the reassortant equine-like G3P[8] strains that have been detected since 2013 in different countries.

First equine-like G3 strains processing a $\mathrm{P}$ [4] were identified in Japan, which exhibited the highest genetic identity with the equine rotavirus strain RVA/Horse-wt/ IND/Erv105/2003-05/G3P[X] [21]. The earliest reported novel equine-like G3 strains processing a P[8] were identified in specimens collected from Australia and Thailand in 2013 [4, 19]. This equine-like G3P[8] has spread around the globe with different detection rates in Australia [4-6], Brazil [7-9], Dominican Republic [10], Germany [11], Hungary [12], Indonesia [13-15], Ireland [16], Italy [17], Japan [18-21], Spain [22], Thailand [23, 24], and USA [25]. In the same time, equine-like G3 strains in combination with a $\mathrm{P}[6]$ had been examined for the first time in samples collected in 2016 in Indonesia $[13,15]$. The findings of equine-like G3 strains combined with various $\mathrm{P}[4], \mathrm{P}[6]$, and $\mathrm{P}[8]$ genotypes indicate the virus diversity and an increased probability of the genome reassortment at the VP4 gene.

In the present study, the equine-like G3P[8] strains, represented by RVA/Human-wt/THA/2CR29/2016 and RVA/Human-wt/THA/2CR41/2016, emerged as the majority RVA gastroenteritis cases. Most recently, equine-like G3P[8] strains with a short RNA pattern, represented by RVA/Human-wt/THA/MS2014-0134/ 2014/G3P[8] and RVA/Human-wt/THA/DBM2016-096/ 2016/G3P[8], were detected at a high frequency in other provinces of Thailand, including Bangkok (80.6\%), Phuket (78.3\%), and Chanthaburi (27.5\%) [24]. This reveals that the equine-like G3P[8] appeared to have emerged around the same time in Northern, Middle, Eastern, and Southern Thailand between 2014 to 2018.

Two RVA vaccines (monovalent Rotarix ${ }^{\mathrm{TM}}$ RV1: G1P[8] and pentavalent RotaTeq ${ }^{\mathrm{TM}}$ RV5: G1, G2, G3, G4, and P[8] have been commercially available since 2011 and are currently added into National Immunization Programs of Thailand since 2019. VP7 and VP8* (a trypsin digestion product of the VP4) contain multiple antigenic epitopes that can induce the production of neutralizing antibodies, which are a main target for vaccine development. Changes or amino acid differences in the antigenic region in these proteins may influence the efficacy of RVA vaccines. In the current survey, the equine-like G3P[8] strains contained many amino acid differences in all antigenic regions compared with the G1, G3, and P[8] strains of RVA vaccines and these amino acid differences were mainly located on the exposed surface of the capsid protein (Figs. 2-3). It has been reported that in Australia the equine-like G3P[8] strains was detected to be more prevalent in areas where Rotarix ${ }^{\mathrm{TM}}$ vaccine was being administered, raising the possibility that RVA vaccine pressure may lead to the selection of novel strains [5, 13]. It remains unclear whether the antigenic difference of the equinelike VP7 and VP8* resulted from the selective pressure by the use of RVA vaccines or is sufficiently prominent to obscure the site from recognition by pre-existing antibodies or effect vaccine efficacy in a subtle way. Taken together, considering that the equine-like G3P[8] rotavirus strains containing several amino acid differences has emerged as one of the most prevalent RVA genotypes in some countries, future RVA vaccine candidates containing the equine-like G3P[8] strain would be the vaccine regionally more specific and provide a secured homotypic protective response to the newly emerging equine-like G3P[8] genotype.

Combination of clinical symptoms between the previous survey in Chiang Rai [23] and this study showed that the severity of symptoms in RVA patients with G3P[8] genotype was similar to that in RVA patients with G9P[8] genotype (Table S1). This is consistent with a survey conducted in Indonesia found no difference of the severity of symptoms between patients associated with the quine-like G3P[8] and the typical human RVA G1 and G3 [13]. In contrast to studies conducted in Australia and Japan, equine-like G3P [8] infections resulted in the most episodes of severe gastroenteritis [4, $20]$.

In conclusion, the emergence of the equine-like G3P [8] strain related to a number of gastroenteritis cases in Thailand, 2016-2018 and other countries indicates the rapid spread of the virus with human and animal gene segments. Information on genetic comparison between the studied strains and the RVA vaccine strains should facilitate the development of a safe and effective RVA vaccine that is efficacious against multiple genotypes 
and variants. Thus, an important priority is to continue identification of the most common $\mathrm{G}$ and $\mathrm{P}$ genotypes and genetic diversity after introduction of RVA vaccines into National Immunization Programs of Thailand since 2019.

\section{Acknowledgments}

This study was partially supported by grants-in-aid from Research and Development Institute, Pibulsongkram Rajabhat University, Thailand. The authors gratefully acknowledge pediatric physicians, nurses, and assistants of Pediatric Unit, Chiangrai Prachanukroh Hospital for fecal specimen collection.

\section{Conflict of Interest}

The authors have no financial conflicts of interest to declare.

\section{References}

1. Tate JE, Burton AH, Boschi-Pinto C, Parashar UD, World Health Organization-Coordinated Global Rotavirus Surveillance Network. 2016. Global, regional, and national estimates of rotavirus mortality in children $<5$ years of age, 2000-2013. Clin. Infect. Dis. 62: S96-S105.

2. Matthijnssens J, Ciarlet M, Rahman M, Attoui $H$, Bányai K, Estes MK, et al. 2008. Recommendations for the classification of group A rotaviruses using all 11 genomic RNA segments. Arch. Virol. 153: 1621-1629.

3. Santos N, Hoshino Y. 2005. Global distribution of rotavirus serotypes/genotypes and its implication for the development and implementation of an effective rotavirus vaccine. Rev. Med. Virol. 15: 29-56.

4. Cowley D, Donato CM, Roczo-Farkas S, Kirkwood CD. 2016. Emergence of a novel equine-like G3P[8] inter-genogroup reassortant rotavirus strain associated with gastroenteritis in Australian children. J. Gen. Virol. 97: 403-410.

5. Roczo-Farkas S, Kirkwood CD, Bines JE, the Australian Rotavirus Surveillance Group. 2016. Australian Rotavirus Surveillance Program annual report, 2015. Commun. Dis. Intell. 40: E527-E538.

6. Roczo-Farkas S, Kirkwood CD, Bines JE, Enteric Virus Group, Murdoch Children Research Institute, Royal Children's Hospital. 2017. Australian Rotavirus Surveillance Program: annual Report, 2016. Commun. Dis. Intell. 41: E455-E471.

7. Luchs A, da Costa AC, Cilli A, Komninakis SCV, Carmona RCC, Boen L, et al. 2019. Spread of the emerging equine-like G3P[8] DS-1-like genetic backbone rotavirus strain in Brazil and identification of potential genetic variants. J. Gen. Virol. 100: 7-25.

8. Guerra SFS, Soares LS, Lobo PS, Penha Júnior ET, Sousa Júnior EC, Bezerra DAM, et al. 2016. Detection of a novel equine-like G3 rotavirus associated with acute gastroenteritis in Brazil. J. Gen.
Virol. 97: 3131-3138.

9. Gutierrez MB, Fialho AM, Maranhão AG, Malta FC, Andrade JDSR, Assis RMS, et al. 2020. Rotavirus A in Brazil: molecular epidemiology and surveillance during 2018-2019. Pathogens. 9:515.

10. Katz EM, Esona MD, Betrapally NS, De La Cruz De Leon LA, Neira YR, Rey GJ, et al. 2019. Whole-gene analysis of inter-genogroup reassortant rotaviruses from the Dominican Republic: emergence of equine-like G3 strains and evidence of their reassortment with locally-circulating strains. Virology 534: 114-431.

11. Pietsch C, Liebert UG. 2018. Molecular characterization of different equine-like $\mathrm{G} 3$ rotavirus strains from Germany. Infect. Genet. Evol. 57: 46-50.

12. Dóró R, Marton S, Bartókné AH, Lengyel G, Agócs Z, Jakab F, et al. 2016. Equine-like G3 rotavirus in Hungary, 2015 - Is it a novel intergenogroup reassortant pandemic strain? Acta. Microbiol. Immunol. Hung. 63: 243-255.

13. Athiyyah AF, Utsumi T, Wahyuni RM, Dinana Z, Yamani LN, Soetjipto, et al. 2019. Molecular epidemiology and clinical features of rotavirus infection among pediatric patients in East Java, Indonesia During 2015-2018: dynamic changes in rotavirus genotypes from equine-like G3 to typical human G1/G3. Front. Microbiol. 10: 940.

14. Cowley D, Nirwati H, Donato CM, Bogdanovic-Sakran N, Boniface $\mathrm{K}$, Kirkwood CD, et al. 2018. Molecular characterisation of rotavirus strains detected during a clinical trial of the human neonatal rotavirus vaccine (RV3-BB) in Indonesia. Vaccine 36: 5872-5878.

15. Utsumi T, Wahyuni RM, Doan YH, Dinana Z, Soegijanto S, Fujii $Y$, et al. 2018. Equine-like G3 rotavirus strains as predominant strains among children in Indonesia in 2015-2016. Infect. Genet. Evol. 61: 224-228.

16. Yandle Z, Coughlan S, Dean J, Tuite G, Conroy A, De Gascun CF. 2020. Group A rotavirus detection and genotype distribution before and after introduction of a National Immunisation Programme in Ireland: 2015-2019. Pathogens 9: 449.

17. Esposito S, Camilloni B, Bianchini S, laniro G, Polinori I, Farinelli E, et al. 2019. First detection of a reassortant G3P[8] rotavirus $A$ strain in Italy: a case report in an 8-year-old child. Virol. J. 16:64.

18. Kikuchi W, Nakagomi T, Gauchan P, Agbemabiese CA, Noguchi A, Nakagomi O, et al. 2018. Detection in Japan of an equine-like $\mathrm{G} 3 \mathrm{P}[8]$ reassortant rotavirus A strain that is highly homologous to European strains across all genome segments. Arch. Virol. 163: 791-794.

19. Komoto $S$, Tacharoenmuang R, Guntapong R, Ide T, Tsuji T, Yoshikawa T, et al. 2016. Reassortment of human and animal rotavirus gene segments in emerging DS-1-Like G1P[8] rotavirus strains. PLoS One 11: e0148416.

20. Komoto S, Ide T, Negoro M, Tanaka T, Asada K, Umemoto M, et al. 2018. Characterization of unusual DS-1-like G3P[8] rotavirus strains in children with diarrhea in Japan. J. Med. Virol. 90: 890898.

21. Malasao $R$, Saito $M$, Suzuki $A$, Imagawa $T$, Nukiwa-Soma $N$, Tohma K, et al. 2015. Human G3P[4] rotavirus obtained in Japan, 2013 , possibly emerged through a human-equine rotavirus reas- 
sortment event. Virus Genes 50: 129-133.

22. Arana A, Montes M, Jere KC, Alkorta M, Gómara Ml, Cilla G. 2016. Emergence and spread of G3P[8] rotaviruses possessing an equine-like VP7 and a DS-1-like genetic backbone in the Basque Country (North of Spain), 2015. Infect. Genet. Evol. 44: 137-144.

23. Chan-It W, Chanta C. 2018. Emergence of G9P[8] rotaviruses in children with acute gastroenteritis in Thailand, 2015-2016. J. Med. Virol. 90: 477-484.

24. Tacharoenmuang R, Komoto $S$, Guntapong R, Upachai S, Singchai $P$, Ide T, et al. 2020. High prevalence of equine-like G3P[8] rotavirus in children and adults with acute gastroenteritis in Thailand. $J$. Med. Virol. 92: 174-186.

25. Perkins C, Mijatovic-Rustempasic S, Ward ML, Cortese MM, Bowen MD. 2017. Genomic characterization of the first equinelike G3P[8] rotavirus strain detected in the United States. Genome Announc. 5: e01341-e01341.

26. Yan H, Nguyen TA, Phan TG, Okitsu S, Li Y, Ushijima H. 2004. Development of RT-multiplex PCR assay for detection of adenovirus and group $A$ and $C$ rotaviruses in diarrheal fecal specimens from children in China. Kansenshogaku Zasshi. 78: 699-709.

27. Gentsch JR, Glass RI, Woods P, Gouvea V, Gorziglia M, Flores J, et al. 1992. Identification of group A rotavirus gene 4 types by polymerase chain reaction. J. Clin. Microbiol. 30: 1365-1373.

28. Gómara MI, Cubitt D, Desselberger U, Gray J. 2001. Amino acid substitution within the VP7 protein of G2 rotavirus strains associated with failure to serotype. J. Clin. Microbiol. 39: 3796-3798.

29. Gómara Ml, Kang G, Gray J. 2004. Rotavirus genotyping: keeping up with an evolving population of human rotaviruses. J. Clin. Virol. 31: 259-265.

30. Esona MD, Gautam R, Tam KI, Williams A, Mijatovic-Rustempasic S, Bowen MD. 2015. Multiplexed one-step RT-PCR VP7 and VP4 genotyping assays for rotaviruses using updated primers. J. Virol. Methods 223: 96-104.

31. Matthijnssens J, Rahman M, Martella V, Xuelei Y, De Vos S, De Leener $\mathrm{K}$, et al. 2006. Full genomic analysis of human rotavirus strain B4106 and lapine rotavirus strain 30/96 provides evidence for interspecies transmission. J. Virol. 80: 3801-3810.

32. Chaimongkol $N$, Khamrin $P$, Suantai $B$, Saikhreang $W$, Thongprachum A, Malasao R, et al. 2012. A wide variety of diarrhea viruses circulating in pediatric patients in Thailand. Clin. Lab. 58: 117123.

33. Chieochansin T, Vutithanachot V, Phumpholsup T, Posuwan N, Theamboonlers A, Poovorawan Y. 2016. The prevalence and genotype diversity of human rotavirus A circulating in Thailand, 2011-2014. Infect. Genet. Evol. 37: 129-136.

34. Jiraphongsa C, Bresee JS, Pongsuwanna $Y$, Kluabwang $P$, Poonawagul U, Arporntip P, et al. 2005. Epidemiology and burden of rotavirus diarrhea in Thailand: Results of sentinel surveillance. J. Infect. Dis. 192: S87-S93.

35. Khamrin P, Peerakome S, Tonusin S, Malasao R, Okitsu S, Mizuguchi
$M$, et al. 2006. Emergence of human G9 rotavirus with an exceptionally high frequency in children admitted to hospital with diarrhea in Chiang Mai, Thailand. J. Med. Virol. 78: 273-280.

36. Khamrin P, Peerakome S, Tonusin S, Malasao R, Okitsu S, Mizuguchi $M$, et al. 2007. Changing pattern of rotavirus $G$ genotype distribution in Chiang Mai, Thailand from 2002 to 2004: decline of G9 and reemergence of G1 and G2. J. Med. Virol. 79: 1775-1782.

37. Khamrin P, Maneekarn N, Malasao R, Nguyen TA, Ishida S, Okitsu S, et al. 2010. Genotypic linkages of VP4, VP6, VP7, NSP4, NSP5 genes of rotaviruses circulating among children with acute gastroenteritis in Thailand. Infect. Genet. Evol. 10: 467-472.

38. Khananurak K, Vutithanachot V, Simakachorn N, Theamboonlers A, Chongsrisawat V, Poovorawan Y. 2010. Prevalence and phylogenetic analysis of rotavirus genotypes in Thailand between 2007 and 2009. Infect. Genet. Evol. 10: 537-545.

39. Maiklang $O$, Vutithanachot $V$, Vutithanachot $C$, Hacharoen $P$, Chieochansin T, Poovorawan Y. 2012. Prevalence of group A genotype human rotavirus among children with dirarrhea in Thailand, 2009-2011. Southeast Asian J. Trop. Med. Public Health 43: 904-916.

40. Sakpaisal P, Silapong S, Yowang A, Boonyasakyothin G, Yuttayong B, Suksawad U, et al. 2019. Prevalence and genotypic distribution of rotavirus in Thailand: a multicenter study. Am. J. Trop. Med. Hyg. 100: $1258-1265$.

41. Theamboonlers A, Bhattarakosol P, Chongsrisawat V, Sungkapalee T, Wutthirattanakowit N, Poovorawan Y. 2008. Molecular characterization of group A human rotaviruses in Bangkok and Buriram, Thailand during 2004-2006 reveals the predominance of G1P[8], G9P[8] and a rare G3P[19] strain. Virus Genes 36: 289298.

42. Yodmeeklin A, Khamrin P, Kumthip K, Malasao R, Ukarapol N, Ushijima $\mathrm{H}$, et al. 2018. Increasing predominance of G8P[8] species $A$ rotaviruses in children admitted to hospital with acute gastroenteritis in Thailand, 2010-2013. Arch. Virol. 163: 21652178.

43. Chan-it W, Thongprachum A, Dey SK, Phan TG, Khamrin P, Okitsu $S$, et al. 2011. Detection and genetic characterization of rotavirus infections in non-hospitalized children with acute gastroenteritis in Japan, 2007-2009. Infect. Genet. Evol. 11: 415-422.

44. Maneekarn N, Khamrin P. 2014. Rotavirus associated gastroenteritis in Thailand. Virusdisease 25: 201-207.

45. Jagai JS, Sarkar R, Castronovo D, Kattula D, McEntee J, Ward H, et al. 2012. Seasonality of rotavirus in South Asia: a meta-analysis approach assessing associations with temperature, precipitation, and vegetation index. PLoS One 7: e38168.

46. Chao DL, Roose A, Roh M, Kotloff KL, Proctor JL. 2019. The seasonality of diarrheal pathogens: A retrospective study of seven sites over three years. PLoS Negl. Trop. Dis. 13: e0007211.

47. Glass RI, Bresee J, Jiang B, Parashar U, Yee E, Gentsch J. Rotavirus and rotavirus vaccines. Adv. Exp. Med. Biol. 582: 45-54. 